\title{
A Single Nucleotide Polymorphism (rs4236480) in TRPV5 Calcium Channel Gene Is Associated with Stone Multiplicity in Calcium Nephrolithiasis Patients
}

\author{
Anas Khaleel, ${ }^{1}$ Mei-Shin Wu, ${ }^{1}$ Henry Sung-Ching Wong, ${ }^{1,2}$ Yu-Wen Hsu, ${ }^{1,2}$ \\ Yii-Her Chou, ${ }^{3,4}$ and Hsiang-Yin Chen ${ }^{1,5}$ \\ ${ }^{1}$ Department of Clinical Pharmacy, School of Pharmacy, Taipei Medical University, Taipei 11031, Taiwan \\ ${ }^{2}$ Master's Program for Clinical Pharmacogenomics and Pharmacoproteomics, School of Pharmacy, \\ Taipei Medical University, Taipei 11031, Taiwan \\ ${ }^{3}$ Department of Urology, College of Medicine, Kaohsiung Medical University, Kaohsiung 80756, Taiwan \\ ${ }^{4}$ Department of Urology, Kaohsiung Medical University Hospital, Kaohsiung 80756, Taiwan \\ ${ }^{5}$ Department of Pharmacy, Wan Fang Hospital, Taipei Medical University, Taipei 11696, Taiwan
}

Correspondence should be addressed to Yii-Her Chou; yihech@gap.kmu.edu.tw and Hsiang-Yin Chen; shawn@tmu.edu.tw

Received 17 December 2014; Revised 31 March 2015; Accepted 17 April 2015

Academic Editor: Grace Kuo

Copyright (C) 2015 Anas Khaleel et al. This is an open access article distributed under the Creative Commons Attribution License, which permits unrestricted use, distribution, and reproduction in any medium, provided the original work is properly cited.

\begin{abstract}
Nephrolithiasis is characterized by calcification of stones in the kidneys from an unknown cause. Animal models demonstrated the functional roles of the transient receptor potential vanilloid member 5 (TRPV5) gene in calcium renal reabsorption and hypercalciuria. Therefore, TRPV5 was suggested to be involved in calcium homeostasis. However, whether genetic polymorphisms of TRPV5 are associated with kidney stone multiplicity or recurrence is unclear. In this study, 365 Taiwanese kidney-stone patients were recruited. Both biochemical data and DNA samples were collected. Genotyping was performed by a TaqMan allelic discrimination assay. We found that a TRPV5 polymorphism (rs4236480) was observed to be associated with stone multiplicity of calcium nephrolithiasis, as the risk of stone multiplicity was higher in patients with the TT+CT genotype than in patients with the CC genotype ( $p=0.0271$ ). In summary, despite the complexity of nephrolithiasis and the potential association of numerous calcium homeostatic absorption/reabsorption factors, TRPV5 plays an important role in the pathogenesis of calcium nephrolithiasis.
\end{abstract}

\section{Introduction}

Nephrolithiasis is a disease caused by calcifications that form stones in the kidney. The major component of these stones is calcium. Nephrolithiasis is a globally common disorder, and the prevalence and incidence of nephrolithiasis have been dramatically increasing worldwide. This phenomenon has placed heavy burdens on national economics and healthcare systems $[1,2]$. Many factors were suggested to be risk factors for nephrolithiasis, such as a family's health history, environmental exposure, for example, to steel manufacturing [3], dietary intake [4-7], a low fluid intake [8], and hypercalciuria [9-12]. Genetic polymorphisms were also reported to be risk factors causing kidney-stone formation and elevating nephrolithiasis recurrence. For example, several genetic variations of the calcium-sensing receptor (CASR) gene were reported to be associated with an increase in recurrent calcium kidney-stone formation [13, 14]. Genetic polymorphisms of Orail, a major subunit of the store-operated calcium channel, were also reported to be associated with nephrolithiasis recurrence [15]. In addition, levels of the vitamin $\mathrm{D}$ receptor $(V D R)$ and its genetic polymorphisms were also indicated to be associated with idiopathic hypercalciuria (IH) $[16,17]$.

The transient receptor potential cation channel, subfamily $\mathrm{V}$, member 5 (TRPV5) is considered the gatekeeper channel for activating calcium transport and reabsorption $[18,19]$. TRPV5 channel was found to be associated with urinary 
calcium (re)absorption in protein expression and channel voltage experiments $[20,21]$. This channel is expressed in the epithelia of renal connecting tubules and distal convoluted tubules, which are major parts of the kidneys involved in calcium homeostasis [22-24]. A previous study indicated that a TRPV5 genetic polymorphism has a conservative calcium effect in African subjects, and an animal model showed that genetic knockdown in mice produced serious hypercalciuria, which is a major risk factor for kidney-stone formation $[18,19]$. Despite the study results of Renkema et al. not supporting a functional role for TRPV5 in the pathogenesis of renal hypercalciuria, they still suggested that the TRPV5 gene should not be excluded as a potential risk factor for hypercalciuria [25].

The goal of this study was to investigate associations of the single-nucleotide polymorphism (SNP), rs4236480, of $T R P V 5$ with the stone number (multiplicity) and recurrence rate in a Taiwanese population.

\section{Material and Methods}

2.1. Patient Recruitment and Study Methods. There were 365 renal-stone patients who fulfilled the diagnostic criteria for nephrolithiasis and were enrolled in this study. Sample collection was approved by the Institutional Review Board of Kaohsiung Medical University Hospital (KMUH) and patients were recruited after signing informed consent. Subjects were excluded if they had a history of renal tubular acidosis, primary and secondary hyperparathyroidism, renal failure, gout, chronic diarrhea, or cancer. We excluded patients with other stone compositions and only recruited patients with idiopathic calcium nephrolithiasis.

Each patient's radiographic and echographic examinations of renal stones were retrieved along with clinical laboratory data, such as age, sex, and family history of nephrolithiasis. Renal-stone samples were obtained by either spontaneous stone passage or surgical intervention. Moreover, in stone number examination, kidney-stone patients with a single stone were allocated to single stone group, while kidney-stone patients having stone numbers exceeding one were allocated to multiple stone group.

The past medical history for renal-stone was retrospectively traced back as far as the patient's medical record was available. Patients who had two or more symptomatic episodes within at least a 6-month interval were classified to the recurrent group; those with only one episode were allocated to the nonrecurrent group. Due to the retrospective nature of the study, some subjects did not have clear past medical history documented in their medical records. Those patients who did not have clear documentations to classify them into either single or recurrent groups were excluded from the analysis for recurrence.

2.2. DNA Extraction. DNA was derived from whole-blood samples which were collected from patients. Blood cells were first treated with $0.5 \%$ sodium dodecylsulfate lysis buffer and then with protease $\mathrm{K}(1 \mathrm{mg} / \mathrm{mL})$ for $4 \mathrm{~h}$ at $60^{\circ} \mathrm{C}$ to digest nuclear proteins. A Gentra (QIAGEN, Valencia, CA)
TABLE 1: Basic characteristics of patients with nephrolithiasis.

\begin{tabular}{lc}
\hline Characteristics & Nephrolithiasis subjects \\
\hline Number of subjects & 365 \\
Sex: male, no (\%) & $250(68.5 \%)$ \\
Age (years) & $54.3 \pm 12.0$ \\
Urine data & \\
$\quad$ Uric acid $(\mathrm{mg} / \mathrm{dL})$ & $40.5 \pm 23.7$ \\
Calcium $(\mathrm{mg} / \mathrm{dL})$ & $10.1 \pm 7.4$ \\
Creatinine $(\mathrm{mg} / \mathrm{dL})$ & $86.3 \pm 56.6$ \\
Serum data & \\
$\quad$ Uric acid $(\mathrm{mg} / \mathrm{dL})$ & $6.5 \pm 1.7$ \\
$\quad$ Calcium $(\mathrm{mg} / \mathrm{dL})$ & $8.9 \pm 0.7$ \\
Creatinine $(\mathrm{mg} / \mathrm{dL})$ & $1.2 \pm 0.9$ \\
\hline
\end{tabular}

extraction kit and 70\% alcohol precipitation were used for DNA extraction by a method described in the previous study [26].

2.3. Genotyping for TRPV5 Polymorphisms. The nonsynonymous SNP, rs4236480, of TRPV5 was selected as the genotyping target. The minor allele frequency of this SNP is $10 \%$ among a Han Chinese population from Beijing, China, based on the HapMap project database (http://hapmap .ncbi.nlm.nih.gov/) [27]. Functionally this SNP represents a nonsynonymous polymorphism in intracellular $\mathrm{N}$ - and Ctails [25]. Genotyping was performed using the TaqMan allelic discrimination assay (Applied Biosystems, Foster City, $\mathrm{CA}$ ). In short, a polymerase chain reaction (PCR) using a 96-well microplate with an ABI 9700 thermal cycler (Applied Biosystems) was performed for the purpose of target sequence enlargement. After the PCR, the fluorescence was measured and analyzed using system SDS software version 1.2.3 (Applied Biosystems). We followed the protocol which was previously reported [26].

2.4. Statistical Analysis. Statistical software R (version 3.0.3) and $\mathrm{R}$ package Bioconductor $[28,29]$ were used for data cleaning and statistical analyses. Genotyping and allelic frequencies between phenotypes were statistically analyzed by the likelihood ratio test. Each $p$ value was adjusted for age and sex. A $p$ value of $<0.05$ was considered statistically significant.

\section{Results}

3.1. Demographic and Clinical Features of Stone-Bearing Patients. We recruited 365 kidney-stone patients for this study. The mean age of patients was $54.3 \pm 12.0$ (standard deviation) years, and $68.5 \%$ of patients were male (Table 1 ). As shown in the HapMap project database, rs 4236480 of TRPV5 has a $10 \%$ minor allelic frequency among a Han Chinese population from Beijing, China [27]. A graphical outline of the rs 4236480 location is shown in Figure 1. nificantly Associated with Stone Multiplicity in Calcium 


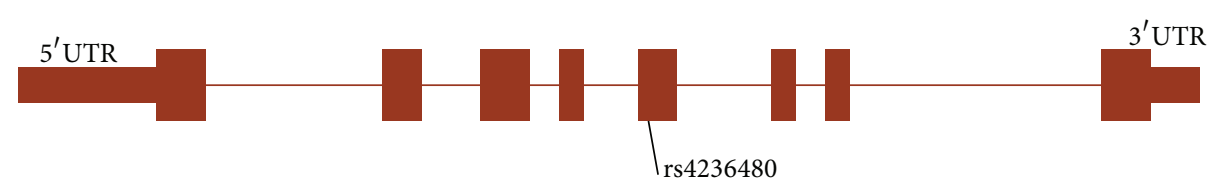

FIGURE 1: Graphical overview of the genotyped human TRPV5 gene.

TABLE 2: Association analysis of TRPV5 rs4236480 and kidney stone multiplicity.

\begin{tabular}{|c|c|c|c|c|c|c|c|}
\hline \multirow{2}{*}{ SNP } & \multirow{2}{*}{ Genotype } & \multicolumn{2}{|c|}{ Multiplicity (\%) } & \multirow{2}{*}{ Genotype $p$} & \multirow{2}{*}{ Dominant $p$} & \multirow{2}{*}{ Recessive $p$} & \multirow{2}{*}{ Log-additive $p$} \\
\hline & & Multiple stone & Single stone & & & & \\
\hline \multirow{3}{*}{ rs 4236480} & TT & $2(2.8)$ & $0(0.0)$ & & & & \\
\hline & CT & $34(47.9)$ & $19(30.2)$ & $0.0372^{*}$ & $0.0271^{*}$ & 0.1125 & $0.0167^{*}$ \\
\hline & $\mathrm{CC}$ & $35(49.3)$ & $44(69.8)$ & & & & \\
\hline
\end{tabular}

The $p$ value was adjusted for sex and age.

${ }^{*}$ The $p$ value was statistically significant.

TABLE 3: Association analysis of TRPV5 rs4236480 and kidney stone recurrence.

\begin{tabular}{|c|c|c|c|c|c|c|c|}
\hline \multirow{2}{*}{ SNP } & \multirow{2}{*}{ Genotype } & \multicolumn{2}{|c|}{ Recurrence (\%) } & \multirow{2}{*}{ Genotype $p$} & \multirow{2}{*}{ Dominant $p$} & \multirow{2}{*}{ Recessive $p$} & \multirow{2}{*}{ Log-additive $p$} \\
\hline & & Yes & No & & & & \\
\hline \multirow{3}{*}{ rs 4236480} & TT & $1(1.9)$ & $2(2.9)$ & & & & \\
\hline & CT & $20(37.7)$ & 19 (27.9) & 0.6502 & 0.5016 & 0.6284 & 0.6462 \\
\hline & $\mathrm{CC}$ & $32(60.4)$ & 47 (69.1) & & & & \\
\hline
\end{tabular}

The $p$ value was adjusted for sex and age.

Nephrolithiasis Patients. In order to investigate the association between stone multiplicity and genotypes, we further examined whether the TRPV5 SNP rs4236480 genotypes were associated with the stone number of calcium nephrolithiasis patients. The $\mathrm{T}$ allele of the rs4236480 polymorphism is a risk allele that appeared to have a dominant genetic effect upon the $\mathrm{C}$ allele (Table 2). The risk for stone multiplicity was higher in patients who carried at least one minor allele than in the wild-type group, and a significant association between rs4236480 and kidney-stone multiplicity was observed (genotype model, $p=0.0372$; dominant model, $p=0.0271 ; \log$ additive model, $p=0.0167$ ) (Table 2 ).

3.3. Lack of an Association of the TRPV5 Genetic Polymorphism (rs4236480) with the Risk of Stone Recurrence in Patients with Calcium Nephrolithiasis. We suspected that SNP genotypes (of rs4236480) were associated with stone recurrence in calcium nephrolithiasis patients. However, the association of genotypes with stone recurrence of calcium nephrolithiasis did not reach statistical significance (Table 3).

3.4. No Association between the rs4236480 Polymorphism of TRPV5 and Biochemical Data of Nephrolithiasis Patients. To better understand the interrelation between rs 4236480 and clinical manifestation risk factors, biochemical data of serum/urine levels of calcium, uric acid, and creatinine were analyzed using urine samples obtained from patients on the spot and with creatinine correction. No significance was found among these clinical parameters (Table 4), and a subgroup analysis also produced no significant results (Table 5).

\section{Discussion}

We recruited 365 nephrolithiasis patients to conduct an association study between the TRPV5 genetic polymorphism, rs4236480, and stone multiplicity/recurrence in calcium nephrolithiasis patients. We found a significant association between rs4236480 and stone multiplicity. To our knowledge, this is the first study to report a significant association of the TRPV5 genetic polymorphism, rs4236480, with the types of kidney-stone multiplicity in Taiwanese calcium nephrolithiasis patients.

Genetic and environmental risk factors influence body levels of calcium ions considerably and thus contribute to calcium nephrolithiasis $[30,31]$. Calcium is the predominant constituent of kidney-stone precipitates, as nearly $80 \%$ of stones are made of calcium, either mostly calcium oxalate or less frequently calcium phosphate [32-34]. High levels of urinary calcium are predominantly urine chemical aberrations in recurrent kidney-stone formers. Subsequently, hypercalciuria is considered to be the main risk factor for renal-stone formation. A family history of nephrolithiasis increases an individual's predisposition for a higher risk of kidney-stone formation $[35,36]$, which substantially implies a susceptibility to genetic causes. Polymorphisms in multiple genes have been recently reported to be associated with the renal stone formation, that include calcium-sensing receptor $(C A S R)$, vitamin D receptor (VDR), and osteopontin $(O P N)$, 


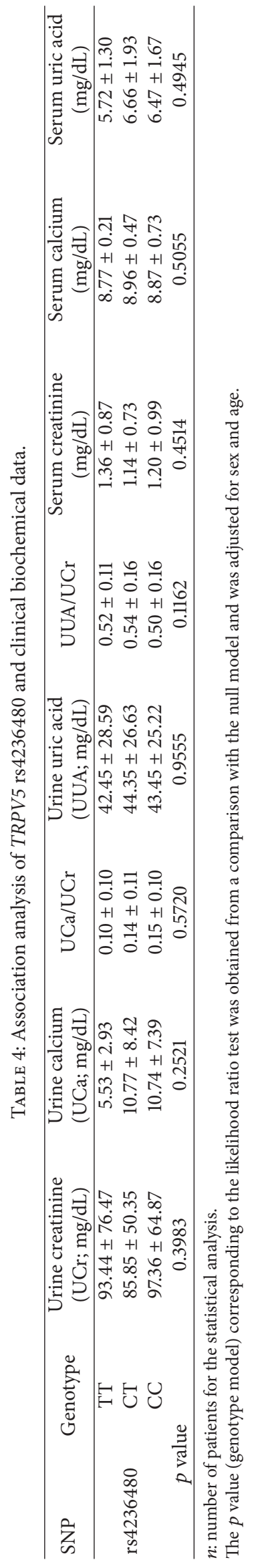


TABLE 5: Association analysis of TRPV5 rs4236480 and subgroups of clinical biochemical data.

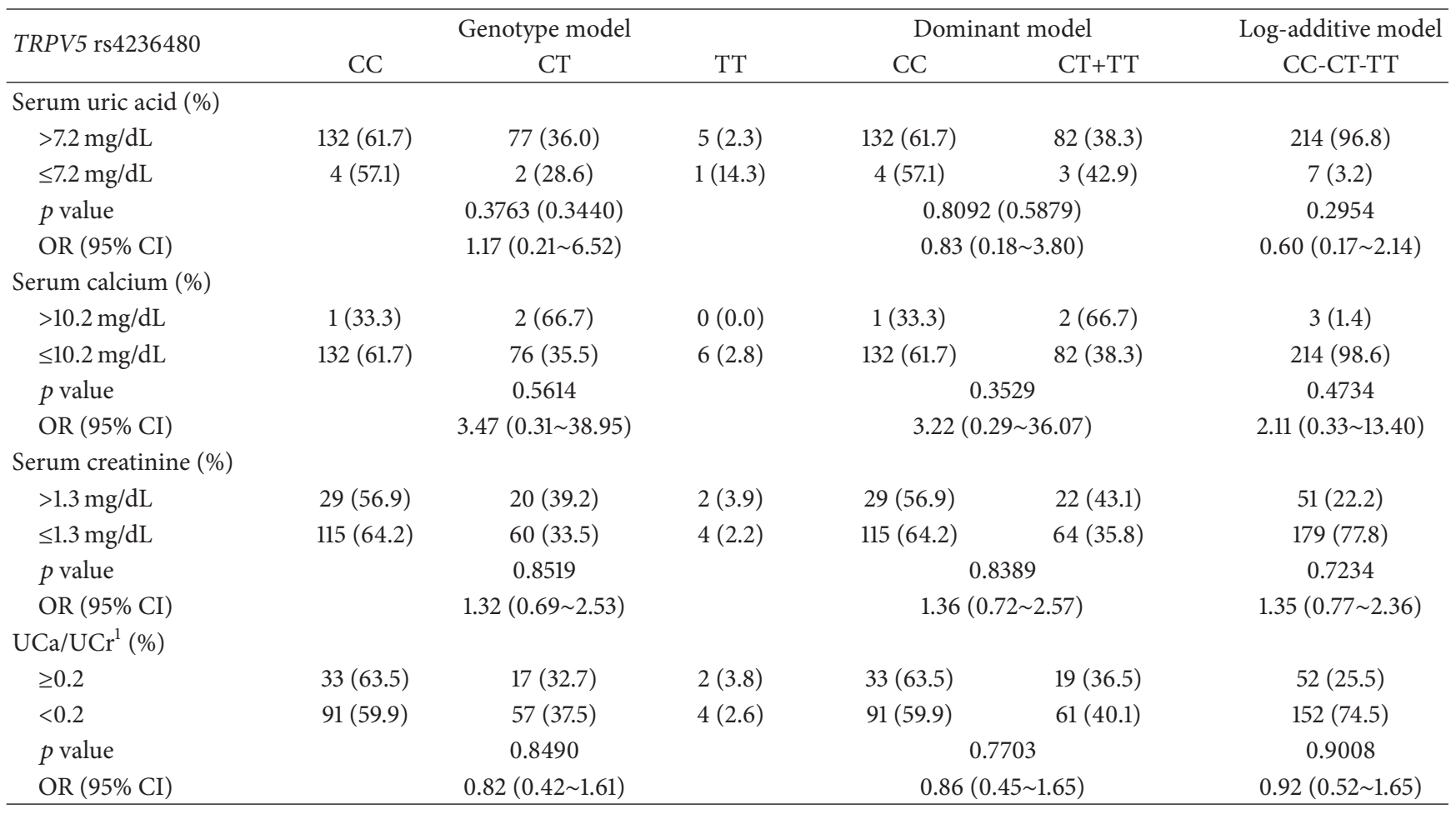

The $p$ value corresponding to the likelihood ratio test was obtained from a comparison with the null model and was adjusted for sex and age.

${ }^{1} \mathrm{UCa} / \mathrm{UCr}$, urinary calcium-creatinine ratio.

OR: odds ratio; CI: confidence interval.

inositol 1,4,5-trisphosphate (IP3) 3-kinase C (ITPKC), and ORAI1 and claudin 14 (CLDN14) genes [15, 26, 37].

TRPV5 is expressed in renal tubules and is regarded as a gatekeeper for calcium ion reabsorption [38, 39]. TRPV5 knockdown mice exhibited severe hypercalciuria [18]. This gene downregulates urinary reabsorption of calcium and exaggerates urinary excretion of calcium in genetic hypercalciuric stone-forming rats [21]. In a previous study, DNA sequence analysis of the coding region of TRPV5 showed a novel T-to-C transition in codon 682 of TRPV5, mutating a conserved serine to a proline (S682P) [40]. In the same report, after comparing wild-type mice to heterozygous and homozygous mutant mice, there was evidence of hypercalciuria, polyuria, and a more-acidic urinary $\mathrm{pH}[40]$.

Limited clinical studies about the association between TRPV5 genotypes and hypercalciuria are available. The first report investigated four TRPV5 polymorphisms in Americans of African descent, where four nonsynonymous SNPs, A8V, R154H (rs4236480), A563T, and L712F, were investigated [19]. Only one variation was found to be significant: A563T of TRPV5 inferred a higher calcium ion reabsorption ability among African Americans, giving people of African descent a conservative superiority for calcium influx over other ethnicities [19]. Another report with 20 French hypercalciuria patients found nonsignificant functional impacts of three TRPV5 SNPs (A8V, R154H, and A561T) [25] compared to wild-type normal TRPV5 [25]. However, the limited set of human subjects' assigned may cause false-negative results. Our results suggest the role of gene polymorphism in increasing the presence of multiple stone numbers in patients carrying this nonsynonymous variation. The results may support a role of TRPV5 in the development of calcium nephrolithiasis in Taiwanese population.

There were a few limitations to this study. First, we observed no association between the rs4236480 polymorphism and increased serum and/or urine levels of calcium in this cohort of Taiwanese patients. Recent genome wide studies have shown how tremendous cumulative factors contribute to serum/urine calcium concentration. O'Seaghdha et al. provided evidence that indicated several polymorphisms in vital genes for calcium homeostasis [41, 42]. Due to polygenic nature of calcium nephrolithiasis, it is difficult to detect a genetic association between one gene and urine/serum calcium concentration with a modest sample size. Additionally, the analysis for stone recurrence generated a null result. It might be, however, a false-negative result from our recurrence analysis due to the limitation of retrospective data collection. A conceivable justification for our current results was the difference in the genetic context of the cohort population and the possible polygenic complexity of nephrolithiasis disease.

\section{Conclusions}

In conclusion, our results indicated a significant association between rs4236480 and kidney-stone multiplicity and 
showed that rs4236480 is a relevant susceptibility indicator for stone multiplicity in nephrolithiasis patients.

\section{Conflict of Interests}

The authors declare that there is no conflict of interests regarding the publication of this paper.

\section{Acknowledgment}

This work was supported by a research Grant from the National Science Council Taiwan (NSC 102-2320-B-038-021) and from Wan Fang Hospital, Taipei Medical University (101WF-EVA-04).

\section{References}

[1] O. W. Moe, "Kidney stones: pathophysiology and medical management," The Lancet, vol. 367, no. 9507, pp. 333-344, 2006.

[2] V. Romero, H. Akpinar, and D. G. Assimos, "Kidney stones: a global picture of prevalence, incidence, and associated risk factors," Reviews in Urology, vol. 12, no. 2-3, pp. e86-e96, 2010.

[3] L. Atan, C. Andreoni, V. Ortiz et al., "High kidney stone risk in men working in steel industry at hot temperatures," Urology, vol. 65 , no. 5 , pp. 858-861, 2005.

[4] G. Bihl and A. Meyers, "Recurrent renal stone diseaseadvances in pathogenesis and clinical management," Lancet, vol. 358, no. 9282, pp. 651-656, 2001.

[5] D. S. Goldfarb, "Prospects for dietary therapy of recurrent nephrolithiasis," Advances in Chronic Kidney Disease, vol. 16, no. 1, pp. 21-29, 2009.

[6] S. R. Khan, P. A. Glenton, and K. J. Byer, "Dietary oxalate and calcium oxalate nephrolithiasis," Journal of Urology, vol. 178, no. 5, pp. 2191-2196, 2007.

[7] E. N. Taylor, M. J. Stampfer, and G. C. Curhan, "Dietary factors and the risk of incident kidney stones in men: new insights after 14 years of follow-up," Journal of the American Society of Nephrology, vol. 15, no. 12, pp. 3225-3232, 2004.

[8] L. Borghi, T. Meschi, F. Amato, A. Briganti, A. Novarini, and A. Giannini, "Urinary volume, water and recurrences in idiopathic calcium nephrolithiasis: a 5-year randomized prospective study," Journal of Urology, vol. 155, no. 3, pp. 839843, 1996

[9] M. B. Damasio, F. Massarino, F. Durand et al., "Prevalence of fasting hypercalciuria associated with increased citraturia in the ambulatory evaluation of nephrolithiasis," Journal of Nephrology, vol. 18, no. 3, pp. 262-266, 2005.

[10] J. Tessier, M. Petrucci, M.-L. Trouvé et al., "A family-based study of metabolic phenotypes in calcium urolithiasis," Kidney International, vol. 60, no. 3, pp. 1141-1147, 2001.

[11] S.-G. Wang, D.-L. Hu, Q.-L. Xi et al., "Expression of CalbindinD28k in genetic hypercalciuric stone-forming rats kidney and its role in pathogenesis of idiopathic hypercalciuria," Zhonghua Yi Xue Za Zhi, vol. 88, no. 20, pp. 1422-1424, 2008.

[12] V. Yoon, B. Adams-Huet, K. Sakhaee, and N. M. Maalouf, "Hyperinsulinemia and urinary calcium excretion in calcium stone formers with idiopathic hypercalciuria," Journal of Clinical Endocrinology and Metabolism, vol. 98, no. 6, pp. 2589-2594, 2013.
[13] Y.-H. Chou, P. Y. Woon, W.-C. Chen et al., "A genetic polymorphism (rs17251221) in the calcium-sensing receptor gene (CASR) is associated with stone multiplicity in calcium nephrolithiasis," PLoS ONE, vol. 6, no. 9, Article ID e25227, 2011.

[14] N. Shakhssalim, B. Kazemi, A. Basiri et al., "Association between calcium-sensing receptor gene polymorphisms and recurrent calcium kidney stone disease: a comprehensive gene analysis," Scandinavian Journal of Urology and Nephrology, vol. 44, no. 6, pp. 406-412, 2010.

[15] Y.-H. Chou, S.-H. H. Juo, Y.-C. Chiu et al., "A polymorphism of the ORAI1 gene is associated with the risk and recurrence of calcium nephrolithiasis," The Journal of Urology, vol. 185, no. 5, pp. 1742-1746, 2011.

[16] M. J. Favus, A. J. Karnauskas, J. H. Parks, and F. L. Coe, "Peripheral blood monocyte vitamin D receptor levels are elevated in patients with idiopathic hypercalciuria," Journal of Clinical Endocrinology and Metabolism, vol. 89, no. 10, pp. 49374943, 2004.

[17] C.-C. Liu, C.-H. Huang, W.-J. Wu et al., "Association of vitamin $D$ receptor (Fok-I) polymorphism with the clinical presentation of calcium urolithiasis," BJU International, vol. 99, no. 6, pp. 1534-1538, 2007.

[18] J. G. J. Hoenderop, J. P. T. M. Van Leeuwen, B. C. J. Van Der Eerden et al., "Renal $\mathrm{Ca}^{2+}$ wasting, hyperabsorption, and reduced bone thickness in mice lacking TRPV5," Journal of Clinical Investigation, vol. 112, no. 12, pp. 1906-1914, 2003.

[19] T. Na, W. Zhang, Y. Jiang et al., "The A563T variation of the renal epithelial calcium channel TRPV5 among African Americans enhances calcium influx," The American Journal of PhysiologyRenal Physiology, vol. 296, no. 5, pp. F1042-F1051, 2009.

[20] D. Müller, J. G. J. Hoenderop, R. Vennekens et al., "Epithelial $\mathrm{Ca}^{2+}$ channel (ECAC1) in autosomal dominant idiopathic hypercalciuria," Nephrology Dialysis Transplantation, vol. 17, no. 9, pp. 1614-1620, 2002.

[21] S. Wang, D. Hu, Q. Xi et al., "The expression and implication of TRPV5, calbindin-D28k and NCX1 in idiopathic hypercalciuria," Journal of Huazhong University of Science and Technology-Medical Science, vol. 28, no. 5, pp. 580-583, 2008.

[22] T. Nijenhuis, J. G. J. Hoenderop, A. W. C. M. van der Kemp, and R. J. M. Bindels, "Localization and regulation of the epithelial $\mathrm{Ca}^{2+}$ channel TRPV6 in the kidney," Journal of the American Society of Nephrology, vol. 14, no. 11, pp. 2731-2740, 2003.

[23] J.-B. Peng, "TRPV5 and TRPV6 in transcellular $\mathrm{Ca}^{2+}$ transport: regulation, gene duplication, and polymorphisms in African populations," Advances in Experimental Medicine and Biology, vol. 704, pp. 239-275, 2011.

[24] V. M. Radhakrishnan, R. Ramalingam, C. B. Larmonier et al., "Post-translational loss of renal trpv5 calcium channel expression, $\mathrm{Ca}^{2+}$ wasting, and bone loss in experimental colitis," Gastroenterology, vol. 145, no. 3, pp. 613-624, 2013.

[25] K. Y. Renkema, K. Lee, C. N. Topala et al., “TRPV5 gene polymorphisms in renal hypercalciuria," Nephrology Dialysis Transplantation, vol. 24, no. 6, pp. 1919-1924, 2009.

[26] W.-C. Kan, Y.-H. Chou, S.-J. Chiu et al., "Study of the association between ITPKC genetic polymorphisms and calcium nephrolithiasis," BioMed Research International, vol. 2014, Article ID 397826, 6 pages, 2014.

[27] C. W. Schmidt, "HapMap: building a database with blocks," EHP Toxicogenomics, vol. 111, no. 1, p. A16, 2003.

[28] R. C. Gentleman, V. J. Carey, D. M. Bates et al., "Bioconductor: open software development for computational biology and bioinformatics," Genome biology, vol. 5, article R80, 2004. 
[29] R Development Core Team, R: A Language and Environment for Statistical Computing, The R Foundation for Statistical Computing, Vienna, Austria, 2014.

[30] S. Aruga and Y. Honma, "Renal calcium excretion and urolithiasis," Clinical Calcium, vol. 21, no. 10, pp. 1465-1472, 2011.

[31] A. E. Stevenson, W. G. Robertson, and P. Markwell, "Risk factor analysis and relative supersaturation as tools for identifying calcium oxalate stone-forming dogs," Journal of Small Animal Practice, vol. 44, no. 11, pp. 491-496, 2003.

[32] A. Trinchieri, "Epidemiology of urolithiasis," Archivio Italiano di Urologia, Andrologia, vol. 68, no. 4, pp. 203-249, 1996.

[33] M. Marangella, P. G. Daniele, M. Ronzani, S. Sonego, and F. Linari, "Urine saturation with calcium salts in normal subjects and idiopathic calcium stone-formers estimated by an improved computer model system," Urological Research, vol. 13, no. 4, pp. 189-193, 1985.

[34] B. T. Murphy and L. N. Pyrah, "The composition, structure, and mechanisms of the formation of urinary calculi," British Journal of Urology, vol. 34, pp. 129-159, 1962.

[35] G. C. Curhan, W. C. Willett, E. B. Rimm, and M. J. Stampfer, "Family history and risk of kidney stones," Journal of the American Society of Nephrology, vol. 8, no. 10, pp. 1568-1573, 1997.

[36] M. Jaggi, Y. Nakagawa, L. Zipperle, and B. Hess, “TammHorsfall protein in recurrent calcium kidney stone formers with positive family history: abnormalities in urinary excretion, molecular structure and function," Urological Research, vol. 35, no. 2, pp. 55-62, 2007.

[37] G. Vezzoli, A. Terranegra, T. Arcidiacono, and L. Soldati, "Genetics and calcium nephrolithiasis," Kidney International, vol. 80, no. 6, pp. 587-593, 2011.

[38] S. Boros, R. J. M. Bindels, and J. G. J. Hoenderop, "Active $\mathrm{Ca}^{2+}$ reabsorption in the connecting tubule," Pflugers Archiv European Journal of Physiology, vol. 458, no. 1, pp. 99-109, 2009.

[39] T. Na and J. B. Peng, "TRPV5: a $\mathrm{Ca}^{2+}$ channel for the finetuning of $\mathrm{Ca}^{2+}$ reabsorption," in Handbook of Experimental Pharmacology, vol. 222, pp. 321-357, Springer, 2014.

[40] N. Y. Loh, L. Bentley, H. Dimke et al., "Autosomal dominant hypercalciuria in a mouse model due to a mutation of the epithelial calcium channel, TRPV5," PLoS ONE, vol. 8, no. 1, Article ID e55412, 2013.

[41] C. M. O’Seaghdha, H. Wu, Q. Yang et al., "Meta-analysis of genome-wide association studies identifies six new Loci for serum calcium concentrations," PLoS Genetics, vol. 9, no. 9, Article ID e1003796, 2013.

[42] C. M. O’Seaghdha, Q. Yang, N. L. Glazer et al., "Common variants in the calcium-sensing receptor gene are associated with total serum calcium levels," Human Molecular Genetics, vol. 19, no. 21, Article ID ddq342, pp. 4296-4303, 2010. 


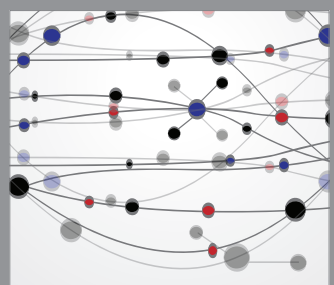

The Scientific World Journal
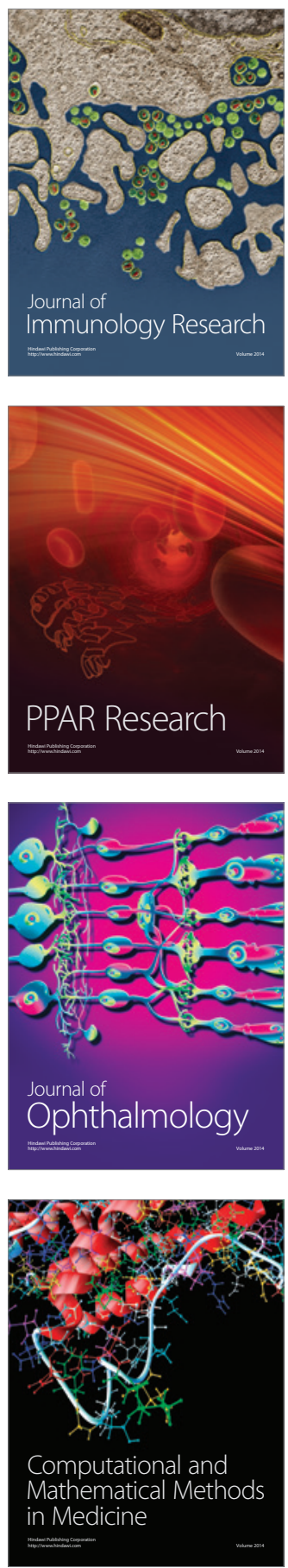

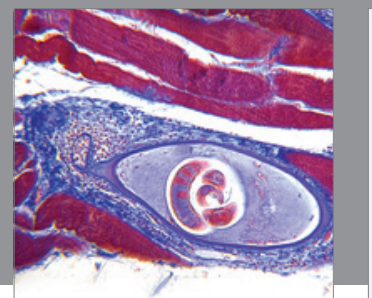

Gastroenterology

Research and Practice
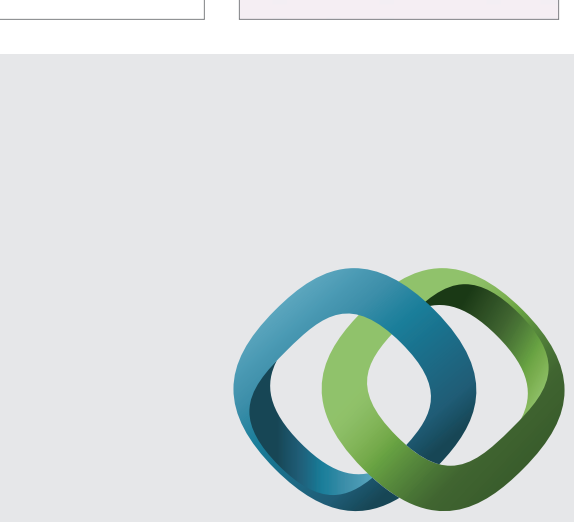

\section{Hindawi}

Submit your manuscripts at

http://www.hindawi.com
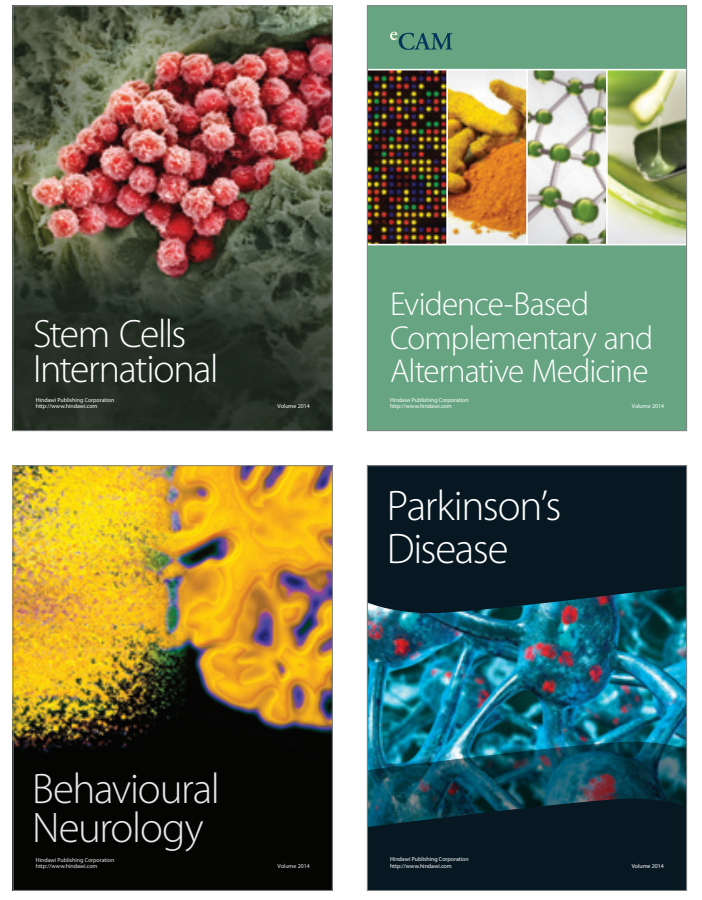
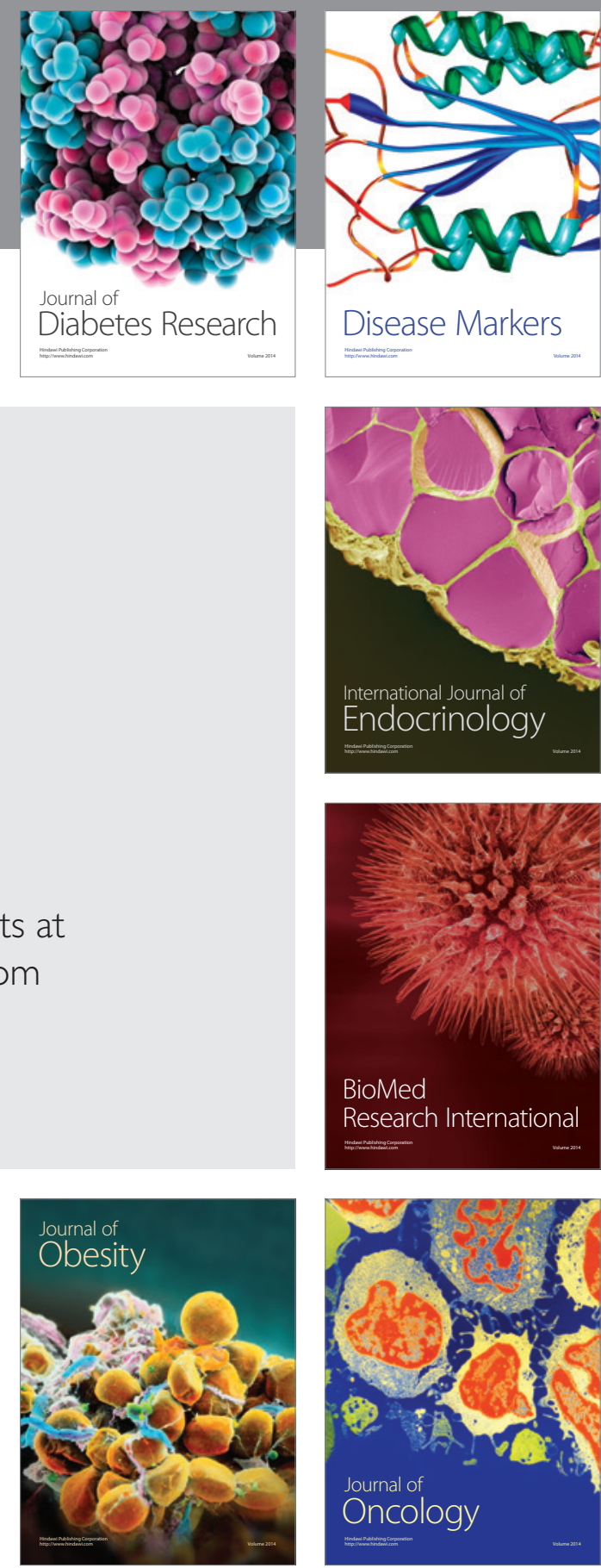

Disease Markers
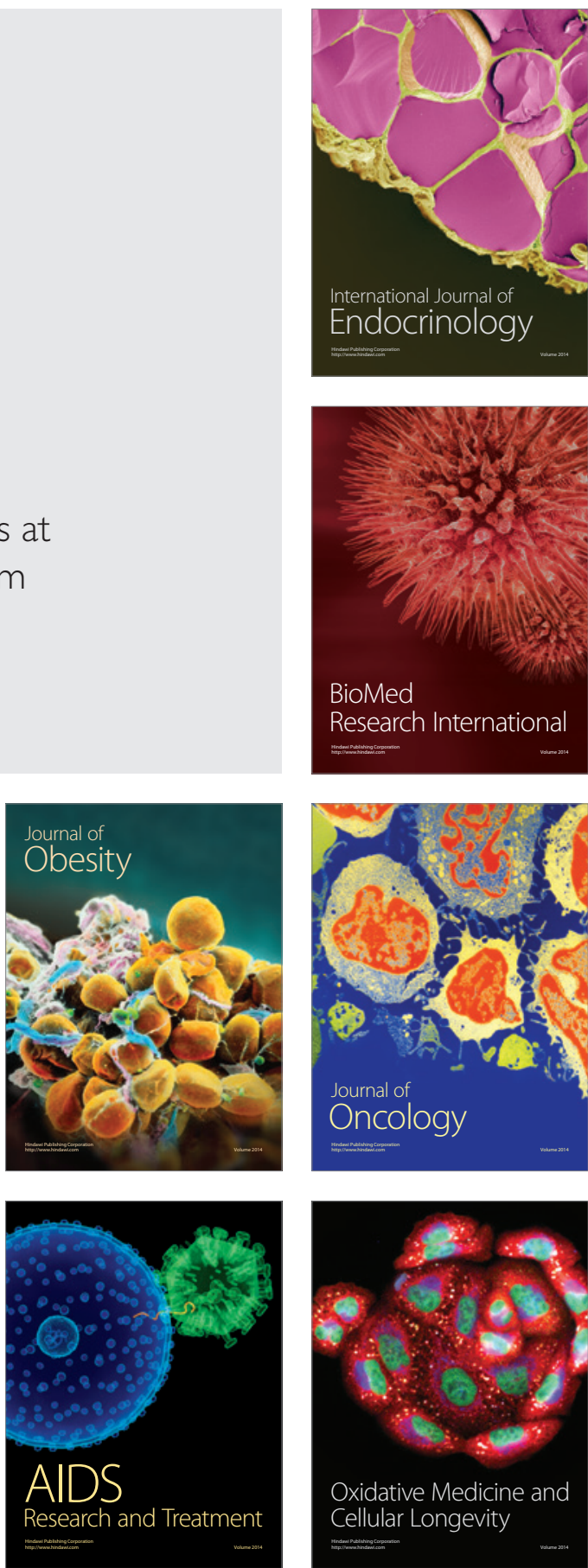